\title{
Rationale and Design of the Lead EvaluAtion for Defibrillation and Reliability (LEADR) Study: Safety and Efficacy of a Novel ICD Lead Design
}

\author{
George Crossley $^{1}$, Prashanthan Sanders ${ }^{2}$, Paolo De Filippo ${ }^{3}$, Khaldoun Tarakji ${ }^{4}$, Bert \\ Hansky $^{5}$, Maully Shah ${ }^{6}$, Pamela Mason ${ }^{7}$, Baerbel Maus ${ }^{8}$, and Keith Holloman ${ }^{9}$ \\ ${ }^{1}$ Vanderbilt University Medical Center \\ ${ }^{2}$ Royal Adelaide Hospital Libraries \\ ${ }^{3}$ Aziende Socio Sanitarie Territoriale Papa Giovanni XXIII \\ ${ }^{4}$ Cleveland Clinic \\ ${ }^{5}$ Städtische Kliniken Bielefeld Germany \\ ${ }^{6}$ The Children's Hospital Philadelphia PA USA \\ ${ }^{7}$ University of Virginia Medical Center \\ ${ }^{8}$ Bakken Research Center Medtronic Inc Maastricht The Netherlands \\ ${ }^{9}$ Medtronic Inc Mounds View Minnesota
}

December 29, 2021

\begin{abstract}
Background: Implantable cardioverter defibrillators (ICD) are indicated for primary and secondary prevention of sudden cardiac arrest. Despite enhancements in design and technologies, the ICD lead is the most vulnerable component of the ICD system and failure of ICD leads remains a significant clinical problem. A novel, small diameter, lumenless, catheter delivered, defibrillator lead was developed with the aim to improve long term reliability. Methods and Results: The Lead Evaluation for Defibrillation and Reliability (LEADR) study is a multi-center, single-arm, Bayesian, adaptive design, pre-market interventional pivotal clinical study. Up to 60 study sites from around the world will participate in the study. Patients indicated for a de novo ICD will undergo defibrillation testing at implantation and clinical assessments at baseline, implant, pre-hospital discharge, 3 months, 6 months, and every 6 months thereafter until official study closure. Patients will participate for a minimum of 18 months to approximately 3 years. Fracture-free survival will be evaluated using a Bayesian statistical method that incorporates both virtual patient data (combination of bench testing to failure with in-vivo use condition data) with clinical patients. The clinical subject sample size will be determined using decision rules for number of subject enrollments and follow-up time based upon the observed number of fractures at certain time points in the study. The adaptive study design will therefore result in a minimum of 500 and a maximum of 900 patients enrolled. Conclusion: The LEADR Clinical Study was designed to efficiently provide evidence for short- and long-term safety and efficacy of a novel lead design using Bayesian methods including a novel virtual patient approach.
\end{abstract}

\section{Hosted file}

LEADER Design Paper Draft_JCE_10DEC2021 Final GHC.docx available at https://authorea. com/users/451172/articles/551037-rationale-and-design-of-the-lead-evaluation-fordefibrillation-and-reliability-leadr-study-safety-and-efficacy-of-a-novel-icd-leaddesign 